\title{
8 Schlussbetrachtung
}

Die vorliegende Untersuchung hat sich zur Aufgabe gemacht, die Schnittstellen von Predigten, Fürstenspiegeln, Urkunden, hagiographischen Texten, Synodalprotokollen und Kapitularien herauszuarbeiten, um damit einen genaueren Eindruck von gesellschaftlichen Prozessen in Bayern in den ersten Jahrzehnten des 9. Jahrhunderts zu gewinnen. Gerade die Einbettung der Predigttexte in die Umgebung bereits bekannter und gut untersuchter Textcorpora und ihre Zuordnung zu politischen Ereignissen hat sich für die Salzburger Sermones-Sammlung als sehr fruchtbar erwiesen. Um das hier entworfene Bild von der sozialen und politischen Funktion der Predigten zu vervollständigen, müssten die zahlreichen anderen Predigtsammlungen und einzelne Predigten, die in Mischhandschriften eingefügt wurden, ebenfalls einer genaueren Untersuchung unterzogen werden. Erst wenn diese Sammlungen aufgearbeitet werden, kann das Bild über die Funktion der Predigt und von predigtähnlichen Texten in den frühmittelalterlichen Gesellschaften geschärft werden. Das trifft für Sammlungen aus anderen Regionen des Karolingerreiches genauso zu wie für die vielen Kompendien aus dem bayerischen Raum, deren Kontexte im Rahmen der vorliegenden Untersuchung nur skizziert werden konnten. Selbstverständlich muss dabei in Betracht gezogen werden, dass viele Predigten verloren gegangen sind und der vorhandene Bestand unseren Blick auf die Produktion und Verteilung von Predigten verzerrt. Während von Erzbischof Arn nun eine Sermones-Sammlung vorliegt, sind z. B. von den Freisinger Bischöfen vergleichbare eigenständige Kompendien im ersten Viertel des 9. Jahrhunderts nicht überliefert, obwohl durchaus von Predigten des Freisinger Bischof Hittos in den Quellen berichtet wird. Aus Freising sind dafür zahlreiche Urkunden vorhanden, die wiederum in den Salzburger Beständen für das 9. Jahrhundert fehlen. Trotz dieses Ungleichgewichts der Überlieferung sind aber insgesamt deutlich mehr Predigten und Predigtsammlungen aus den frühmittelalterlichen Skriptorien überliefert als beispielsweise historiographische Texte. Sie bieten daher eine Möglichkeit, unser Wissen über die Geschichte des 9. Jahrhunderts zu vergrößern. Gerade weil es sich bei den überlieferten Predigten oft „nur“ um Kompilationen handelt, erlauben sie im Vergleich zu ihren Vorlagen einen genauen Einblick in Auswahlstrategien und die damit verbundenen Aneignungsprozesse für unterschiedliche, fallweise sozial differenzierte oder lokal definierte Gruppen. Aus der historischen Perspektive gebührt der frühmittelalterlichen Predigt nicht zuletzt deshalb ein gleichwertiges Interesse wie ihren spätantiken und hochmittelalterlichen Pendants.

Seit der Spätantike war die Predigt das wichtigste Instrument der Glaubensverkündung. Viele der Prediger dieser Zeit waren noch rhetorisch geschult und ihr Publikum war noch kundig genug, um den tiefsinnigen moralischen und exegetischen Argumenten zu folgen und an den sprachlichen Finessen Gefallen zu finden. Obwohl die Überlieferung schriftlich niedergelegter Predigten nach dem 5. Jahrhundert mit wenigen lokalen Ausnahmen ausdünnte und damit auch eine veränderte Qualität des Predigens einherging, blieb die Predigt nach wie vor ein wichtiges Medium. Ihren 
hohen Stellenwert dokumentieren vor allem hagiographische Quellen, in denen der Erfolg eines Heiligen sehr oft anhand seiner Predigtleistung verdeutlicht wird. Wenn um 800 die Predigt in den Vordergrund rückt und in unterschiedlichen Texten verstärkt thematisiert wird, dann ist dies vor dem Hintergrund und auf Grundlage einer langen und erfolgreichen Tradition zu sehen, die in der Spätantike ihren Ausgangspunkt nahm. Den karolingischen Intellektuellen waren diese Wurzeln bewusst und gerade um 800 waren es die alten christlichen Traditionen und ihre universellen moralischen Werte, die diese Texte für die karolingische Politik so wertvoll machten.

Vor allem mit der Admonitio generalis von 789 wurde die Predigt verstärkt zu einer Angelegenheit des fränkischen Königs erklärt. Seine admonitio wurde sogar als praedicatio begriffen. Die Bischöfe wurden aufgefordert, in ihren Diözesen vermehrt zu predigen und auch ihre Priester zur Predigt zu verpflichten. In den meisten darauf folgenden Kapitularien wurde diese Verpflichtung wiederholt. Zeugnisse dieser Bemühungen stellen die bischöflichen Kapitularien dar, die um 800 erstmals publiziert wurden. Dabei zeigt sich, dass die Voraussetzungen regional sehr unterschiedlich waren. An vielen Orten mussten die Priester überhaupt erst in den Grundlagen des Predigens unterwiesen werden, in anderen konnte man auf Vorkenntnisse aufbauen, wenn auch auf bescheidene. Durch die Notwendigkeit der Unterweisung lassen sich auch vereinzelt Spuren einer Reflexion über Predigtinhalte und Predigttechniken finden. In Salzburg führte dies wahrscheinlich noch zur Zeit Arns zu einer Überarbeitung der Schrift De doctrina christiana des Augustinus. Gleichzeitig finden sich Predigttexte in verschieden gestalteten Handbüchern, die Bischöfe für ihre Visitationsreisen nutzten. Zahlreiche Homiliare und Sermones-Sammlungen, deren Überlieferung seit den letzten Jahren des 8. Jahrhunderts einsetzt, zeugen von einer deutlich eifrigeren Predigttätigkeit in den einzelnen Diözesen, vor allem im bayerischen Raum.

Nicht alle diese Predigttexte haben aber die Laienbevölkerung direkt erreicht. Manche waren für das private Studium bestimmt, andere für den monastischen Gebrauch. Aber selbst dann fanden sie oft einen Weg zu Laien, in dem Sinne, dass selbst Mönche und Kanoniker als Multiplikatoren der Predigtinhalte - allerdings im kleineren Rahmen - dienten.

Die erkennbar verstärkte Produktion und Verbreitung von Predigthandschriften verdeutlicht, dass der Versuch, Glaubenslehre und moralische Unterweisungen möglichst in allen sozialen Schichten des Karolingerreiches fest zu verankern, zumindest ansatzweise Erfolg hatte. Im Idealfall sollten sie die Mitglieder der familiae erreichen. Die Predigten sollten eine gemeinsame Basis sowohl in der weltlichen als auch in der säkularen Sphäre schaffen. Für die monastischen Gemeinschaften wurde durch Paulus Diaconus ein Kompendium von autorisierten Texten zusammengestellt, das eine einheitliche Lektüre im gesamten Reich ermöglichte. Aber das waren im Unterschied zu dem Großteil der Gesellschaft Textgemeinschaften, mit einem für das liturgische Jahr mehr oder weniger gleichgeschalteten (Rezeptions-)Rhythmus. Vergleichbares für die illiterate Laienbevölkerung zu erreichen, war nicht leicht möglich. Hier sollten die wichtigsten Elemente der Glaubenslehre zumindest so verankert 
werden, dass der Einzelne seine Erlösung erreichen konnte. ${ }^{1}$ Musterpredigten in der Volkssprache verdeutlichen, dass ernsthafte Versuche unternommen wurden, dieses Ziel zu erreichen. ${ }^{2}$ Das hatte durchaus mehr mit karolingischer Staatlichkeit zu tun, als es auf den ersten Blick scheinen mag. Denn nur auf Basis der Heilserwartung konnten dem karolingischen Untertanen im Zuge der Vereidigung auf den Kaiser auch die weitreichenden Konsequenzen im Fall eines Eidbruches klargemacht werden. Die moralischen Aspekte des Glaubens standen deshalb im Vordergrund der Predigten und sollten im Idealfall einen Sinn für das gemeinsame große Ganze schaffen, das über ethnische Unterschiede hinauswies und in dessen Zentrum der König bzw. der Kaiser stand. Für Karl den Großen und für die Intellektuellen an seinem Hof war es ein erklärtes Ziel, dieses Bewusstsein im Reich zu schaffen. Für Alkuin war spätestens nach dem Überfall der Wikinger im Frühsommer 793 auf Lindisfarne klar, dass zwischen der unmoralischen Lebensführung der principes, iudices und des gesamten populus und dem Verlust der patria ein Zusammenhang bestand. Da der Brief, in dem er diese Verbindung herstellte, an einen Bischof gerichtet war, vergaß er auch nicht, daran zu erinnern, dass sich die Katastrophe vor allem auch wegen der Untätigkeit (desidia) und Trägheit (pigritia) der bischöflichen Prediger ereignet hatte. ${ }^{3}$

Vor allem über das Medium der Predigt konnte also das Unternehmen, das auf die Moralisierung der Gesellschaft abzielte, erfolgreich durchgeführt werden. Die Predigt hatte den Vorteil, dass man auf eine bereits existierende, mehr oder weniger funktionierende Institution zurückgreifen und diese ausbauen konnte. Daher wurde auch auf die Regelmäßigkeit des Predigens wert gelegt und auf den offenen Zugang zur sonntäglichen Predigt geachtet. Zudem wurden seit den 780er Jahren in regelmäßigen Abständen Versammlungen einberufen, zu deren Anlass Ansprachen und Predigten gehalten wurden. Das betraf sowohl Reichsversammlungen als auch Konzile und sonstige conloquia, die jeweils von Geistlichen und Laien besucht wurden. In Bayern lassen sich um und unmittelbar nach 800 besonders viele Synoden in unterschiedlichen Größenordnungen nachweisen. In einem von Arn von Salzburg verfassten Konzilsordo wird der Stellenwert der Predigt im Rahmen der von ihm einberufenen Synoden besonders deutlich. Von gleicher Bedeutung waren Gerichtstage, bei denen Beschlüsse verlautbart und die in Gerichtsfällen involvierten Personen ermahnt wurden, sowie Versammlungen anlässlich der Vereidigung der Bevölkerung, wie sie 789 oder 802 erfolgten. Das Capitulare missorum (792 vel 786), das wohl 789 erlassen wurde, gibt einen guten Eindruck davon, welche Bevölkerungsteile zusammengerufen wurden. ${ }^{4}$ Somit war im Karolingerreich ein dichtes Netz von Predigten und Reden gespannt, wobei Bayern eine Sonderstellung zukam. Dieses Netz überschnitt sich an

1 Amos, Preaching and the Sermon 52.

2 Wilhelm Scherer, Eine lateinische Musterpredigt aus der zeit Karls des grossen, in: Zeitschrift für deutsches Alterthum 12 (1865) 436-446.

3 Alkuin, Epistola 17, ed. Dümmler 47.

4 Capitulare missorum a 792 vel 786 c. 4, ed. Boretius 67. Zur Datierung siehe Becher, Eid und Herrschaft $78-87$. 
manchen Stellen mit dem Wirkungskreis der missi, die ebenfalls die ganze Gesellschaft, ob Herr oder Knecht, Mann oder Frau, stark oder schwach, reich oder arm, erreichen sollten. ${ }^{5}$

Ein Vorteil der Predigt für die Vermittlung politischer Inhalte lag auch darin, dass durch stetiges Wiederholen der Inhalte moralische Werte nachhaltig eingeschärft werden konnten. ${ }^{6}$ Das war eine Voraussetzung, um eine stabile „vision of community“ im Reich zu etablieren. In Katastrophenfällen, die Unruhe im sozialen Raum erzeugten, konnte auf diese Werte leicht Bezug genommen werden, wie es Karl der Große im Jahr 806 im Zuge seiner Gesetzgebung zur herrschenden Hungersnot tat.

Die meisten Predigten beziehen sich zudem auf das Leben nach dem Tod und fordern das Individuum auf, sich in jeder Situation für die Tugenden und gegen die Sünden zu entscheiden. Diese dem Genre inhärente „Rhetorik der Krise“ eignete sich besonders dafür, politische Inhalte zu vermitteln, zumal die Karolinger selbst vor allem in den Kapitularien eine „Rhetorik der Reform“ pflegten. ${ }^{7}$ Diese Rhetorik versuchte die Kontrolle über die Eliten an den peripheren Gebieten des Reiches dadurch zu erlangen, indem sie Hergebrachtes in Frage stellte, also als reformbedürftig qualifizierte. Insofern überschnitten sich Predigten atmosphärisch und thematisch mit Kapitularien und Synodalbeschlüssen. Viele Predigten nahmen aber auch Themen der Gesetzgebung auf, wie auch ausgesuchte Predigt-Passagen in Konzilsbeschlüsse eingingen. Dabei griffen sowohl Kapitularien, Konzilsbeschlüsse als auch Sermones auf gemeinsame (patristische) Quellen zurück. ${ }^{8}$ In der mündlichen Verbreitung dieser Texte zeigten sich ebenfalls Überschneidungen. In manchen Fällen wurden rechtliche Bestimmungen vom Hof von der Diözesanpriesterschaft (regelmäßig) verlesen und erklärt. In anderen wurden Kapitularien in die Form einer Predigt gebracht und von einem missus vorgetragen. Wenngleich die Aufgabenbereiche von Bischöfen und Grafen getrennt waren, gab es doch auch dabei zahlreiche Gemeinsamkeiten. Viele Bischöfe wurden als missi dominici eingesetzt, wie Arn von Salzburg, und waren deshalb auch mit gerichtlichen Auseinandersetzungen betraut. In den bayerischen Traditionsnotizen erscheint der Salzburger Erzbischof demnach mit unterschiedlichen Bezeichnungen (als archiepiscopus, episcopus, pontifex und missus), ohne dass man daraus ver-

5 Vgl. Arn von Salzburg, Amonitio, ed. Schneider 486.

6 Peter Brown, Autorität und Heiligkeit. Aspekte der Christianisierung des Römischen Reiches (Stuttgart 1998) 95, zitiert James Fentress/Chris Wickham, Social Memory (Oxford/Cambridge-Massachusettes 1992) 51: „Die Stereotypen, in vielen Heiligenleben sich ständig wiederholenden Berichte über bestimmte Ereignisse sollten wir daher in ihrer Tragweite nicht unterschätzen ... dass, ... die dauernde Wiederholung solcher Geschichten uns einen Grundvorrat an Erklärungsmöglichkeiten vermittelt und so unsere Sicht der Realität mitprägt.“

7 Zum Limit der tatsächlich erreichten Ziele vgl. Wallace-Hadrill, The Frankish Church 156; Bullough, Europae pater 90. Siehe auch Fouracre, Carolingian justice 783, Anm. 32.Vgl. Amos, Preaching and the sermon 52.

8 Mordek/Schmitz, Neue Kapitularien 401. Vgl. Predigtsammlung mit Capitulare ecclesiasticum Caroli magni $(805-813)$ c. 5: De avaricia cavenda, ed. Mordek/Schmitz $400 \mathrm{f}$. 
schiedene Funktionen ableiten könnte. ${ }^{9}$ Zugleich waren aber auch die weltlichen Amtsträger für das Seelenheil des populus verantwortlich, wie es einige im Jahr 806 von missi dominici verlautbarten Capitula für die comites um Lüttich verdeutlichen. In diesem Fall mahnten sogar vier namentlich genannte Grafen ihre Standesgenossen. ${ }^{10}$

Einen Eindruck, welche Themen bei einer Mahnrede eines comes angesprochen wurden, vermitteln unterschiedliche Quellen. Zur gleichen Zeit wurden nämlich manche comites, wie Maginharius von Sens und Erich von Friaul, später auch Matfrid von Orléans, dezidiert dazu aufgefordert, die moralische Unterweisung ihrer Untergebenen zu übernehmen. ${ }^{11}$ Als Grundlage sollten durchwegs jene moralischen Traktate dienen, die ihnen gewidmet worden waren. Im Fall von Erich von Friaul zeigt sich, dass sich eine solche Mahnrede inhaltlich kaum von einer Predigt unterschied.

Die thematische Schnittmenge von Kapitularien, Mahnreden und Predigten ergab sich aber schließlich auch daraus, dass Geistliche wie Alkuin von Tours oder Arn von Salzburg, beide Autoren moralischer Traktate, von Mahnschreiben bzw. von Predigten, bei der Gestaltung von Kapitularien wesentlich mitwirkten. Alkuin gilt als der Spiritus rector der Admonitio generalis. ${ }^{12}$ Arn von Salzburg war bei den Reformsynoden 801/2 federführend beteiligt, gesichert ist auch sein Beitrag in Mainz im Jahr 813. ${ }^{13}$

Insofern füllten die Vermittlung von Kapitularien, die von den Grafen erforderte Ermahnung ihrer Untergebenen und die Inhalte zeitgenössischer Predigten gemeinsam und stetig das Repertoire jener moralischen Maßstäbe auf, aus denen die politische Kommunikation dieser Zeit schöpfte. Befeuert wurde dieser Diskurs durch Theodulfs Carmen contra iudices, in dem er die Bestechlichkeit der Richter und der Königsboten schilderte und so zu verstehen gab, dass damit auch die Kommunikationslinien vom Hof in die Regionen bzw. vom König zu seinen Untertanen korrumpiert waren. Aber auch Arn von Salzburg legte bereits 799 Konzepte am Hof vor, die sowohl die Reform der Königsboten, als auch den Korruptionsdiskurs betrafen. Im Zentrum der Reformen standen deshalb vor allem weltliche Amtsträger als Repräsentanten des Staates. Wenngleich viele Reformen nur auf dem Pergament bestanden, sind doch einige angedachten Schritte wie die Einführung der Schöffenverfassung oder der Gebrauch des Inquisitionsverfahrens in Bayern durchgesetzt worden. Die Änderungen betrafen also die Neudefinition von Funktionen jener Amtsträger, die Recht sprachen. Gerade die Reformen von 802 trachteten danach, den weltlichen Amtsträgern nicht nur Rechtsnormen zur Verfügung zu stellen, sondern ihnen auch ein positives Modell für ihre Lebensführung zu präsentieren, das ihre Rolle als Richter miteinschloss. ${ }^{14}$

9 Wolfram, Arn von Salzburg und Karl der Große 28.

10 Capitula ad missis dominicis comites directa, ed. Boretius $183 \mathrm{f}$. Vgl. de Jong, Charlemagne‘s church 107; McKitterick, Charlemagne 218.

11 Agobard, Epistola 10 ad Matfredum, ed. Dümmler 202 f. Noble, Secular Sanctity 14.

12 Siehe Glatthaar (ed.), Die Admonitio generalis Karls des Großen, Einleitung 47 - 63.

13 Siehe Wolfram, Salzburg, Bayern, Österreich 186, mit Hinweis auf Goldberg.

14 Vgl. Innes, Charlemagne, justice and written law 169, mit dem Hinweis auf einen implizieten Vergleich zwischen weltlichem Gesetz und Mönchsregel. 
Um 800 zeichnet sich deshalb eine „Kultur der moralischen Evaluierung“ ab, in deren Zentrum vor allem comites standen. ${ }^{15}$ Grafen wie Magenharius von Sens, ein Chrodgarius $^{16}$ und ein Ardbert ${ }^{17}$ wurden von Alkuin ebenso beraten wie Erich von Friaul und wahrscheinlich der praefectus von Bayern, der comes Gerold. Die meisten suchten den Kontakt zum Angelsachsen, um sich unterweisen zu lassen, wie Erich von Friaul, der im Jahr 795 das Gespräch mit Alkuin in einer kleinen Hütte wahrscheinlich in Aachen suchte. Wie wir aus einem Brief erfahren, hatte der Angelsachse nicht nur für die Gesundheit Erichs gebetet, sondern ihn auch ermahnt, die Gebote Gottes einzuhalten. Nur so würde er bewirken, „dass Gottes hochheiliger Wille dich im Guten lenkt und im Schlechten beschützt. “ Diese Mahnung hat bekanntlich nicht gefruchtet. Aber ein mächtiger Berater wie der Abt von Tours stellte sicher mehr als nur eine spirituelle Autorität dar, die gute Ratschläge gab. Er sicherte auch den Kontakt zum Hof bzw. zu jenen Kreisen, die die moralischen Standards überhaupt erst definierten. ${ }^{18}$ Dazu zählten aber auch spirituelle Autoritäten an den Rändern des Reiches, wie Arn von Salzburg und Paulinus von Aquileia. In seinem Brief an Erich von Friaul verwies Alkuin den comes an den Patriarchen von Aquileia, den er als dessen consiliarius bezeichnet.

Aber auch im lokalen Rahmen lassen sich Spuren eines Diskurses finden, der nach der Eignung für das Amt fragt. Im Jänner 814 übertrug der comes Orendil Besitz in Schammach (im Ebensberger Raum) an Freising unter der Auflage, dass dieser an einen seiner Söhne als Lehen rückverliehen würde, falls sich einer von ihnen als würdig erweisen werde (dignus fuerit), das ministerium comitis zu erlangen. ${ }^{19}$ Orendil empfand die Nachfolge eines seiner Söhne in das Grafenamt keineswegs als selbstverständlich bzw. er fand die „richtigen“ Worte dafür, um diesen Eindruck zu vermitteln. Er selbst hatte das Amt eines comes erst um 806 übernommen. Davor hatte er vier Jahre als iudex gewirkt. Tatsächlich startete seine Karriere als Richter erst als Arn und Audulf an einem Frühsommertag 802 erstmals einen Gerichtstag hielten, an dem sie die bayerischen Eliten von der Etablierung des ständigen Missaticums in Bayern unterrichteten und dabei wahrscheinlich auch die notwendigen moralischen Standards bei den Amtsträgern einmahnten. Orendil, der unmittelbar darauf mit einem der schwierigsten bayerischen Konflikte betraut wurde, scheint das rückhaltlose Vertrauen der missi genossen zu haben. Dass er mit den Ideen der Reformen bzw. mit der

15 Innes, ,A place of discipline’ 75, spricht mit den Worten M. Foucaults über eine am Hof herrschende „technology of the self“.

16 Alkuin, Epistola 224, ed. Dümmler $367 \mathrm{f}$.

17 Alkuin, Epistola 302, ed. Dümmler 460f. Zur Identifizierung Ardberts als ein optimas in Mercia vgl. Bullough, Alcuin and lay virtue 77.

18 Vgl. Alkuin, Epistola 33, ed. Dümmler 74f., an den comes von Sens Magenharius; ders., Epistola 224, ed. Dümmler $367 \mathrm{f}$., an den comes Chrodgarius.

19 TF 313, ed. Bitterauf 268f.; vgl. Hannig, Funktion der „missi dominici“ 286; Stone, Morality and Masculinity 151; Störmer, Früher Adel 2, 458; Kohl, Lokale Gesellschaften 323, Anm. 338; Thomas Zotz, In Amt und Würden. Zur Eigenart „offizieller“ Positionen im früheren Mittelalter, in: Tel Aviver Jahrbuch für deutsche Geschichte 22 (1993) 1-23, hier $12 \mathrm{f}$. 
Rhetorik der Reform vertraut war, bezeugt die Vorbehaltsklausel in seiner Schenkungsurkunde. Für eine individuelle Basis dieser Evaluierung sorgten die zahlreichen Predigten zur Beichte und Buße. Denn dort wurde dem Einzelnen eine Art Innenbeschau nahegelegt.

Die Kultur der Evaluierung war auch von wechselseitigem Nutzen. Laien fragten nach spiritueller Führung und moralischer Unterweisung, wie Wido von der Bretagne und Matfrid von Orléans und konnten so engen Kontakt zu den inneren Zirkeln am Hof halten. In Dhuodas Handbuch für ihren Sohn Wilhelm erkennt man, für wie bedeutsam die Mutter diese Kontakte erachtete. ${ }^{20}$ Der Hof wiederum konnte so die Eliten an sich binden bzw. die Verbreitung der Reformen sichern. ${ }^{21}$ Nur in den wenigsten Fällen kann man nämlich schwere Eingriffe des Hofes in das hierarchische Gefüge lokaler Eliten beobachten. Dafür fehlte es oft an strukturellen Mitteln oder an Durchsetzungskraft. An manchen Orten ist aber im Zuge der gerichtlichen Reformen der Aufstieg einer neuen Elite zu beobachten. Die Karriere des comes Orendil dürfte ein solcher Fall gewesen sein. Meist stammten die Richter aber aus den alten Eliten und passten sich nur den neuen Vorgaben an.

Besonders bedeutsame Amtsträger, vor allem jene, die Schlüsselpositionen an den Rändern des Reiches übernommen hatten, wurden mit größeren moralischen Traktaten versorgt. Wido von der Bretagne empfing die Schrift De virtutibus et vitiis von Alkuin, Erich von Friaul bekam den Liber exhortationis von Paulinus von Aquileia; später verfasste Jonas von Orléans De institutione laicali für den comes Matfrid, der am südlichen Rand des Karolingerreiches kämpfte. ${ }^{22}$ Und Arn von Salzburg stellte für den comes Audulf in Bayern, der auch mit der Erfassung des Ostlandes betraut war, eine Sermones-Sammlung zusammen, nachdem er wahrscheinlich im Frühjahr 802 von Alkuin ermahnt worden war, Audulf zu predigen.

Diese Sammlung stellte eine formale Neuheit dar und fügte sich damit in eine Reihe von anderen Salzburger Eigenheiten ein, die Bayern unter der Herrschaft Karls des Großen als ein „Experimentierfeld“ auswiesen. In diesem Fall wurde eine moralische Mahnschrift nicht in Form eines Traktats vorgelegt, sondern in Predigten gefasst. Damit entsprach diese Sammlung genau den Forderungen dieser Zeit: die admonitio der Grafen wurde mit der praedicatio zusammengeführt. ${ }^{23}$ Schon Alkuin hatte in seinem Traktat De virtutibus et vitiis von einer sermonis series gesprochen und es

20 Dhuoda, Liber manualis 3, 9 und 10, ed. Thiébaux 106-117. Vgl. Nelson, Dhuoda 116.

21 Fouracre, Carolingian justice.

22 Michel Sot, Concordances et discordances entre culture des élites laïques et culture des élites cléricales à l'époque carolingienne: Jonas d'Orléans et Dhuoda, in: La culture du haut moyen âge. Une question d'élites?, ed. François Bougard/Régine Le Jan/Rosamond McKitterick (Collection Haut Moyen Âge, 7, Turnhout 2009) 341-361.

23 Schon Pierre Toubert, La théorie du mariage chez les moralists carolingiens, in: Il matrimonio nella società altomedievale 1 (Settimane di studio del Centro italiano di srudi sull'alto medioevo 24, 1 , Spoleto 1977) 233-285, hier 244, betont, dass es bei den Laienspiegeln keine typische Struktur gab. 
damit in die Nähe der Rede gerückt. ${ }^{24}$ In der Vita Alcuini aus den 820er Jahren wird der Predigtcharakter des Traktats besonders deutlich. Demnach habe Alkuin ad Widonem comitem omelias de principalibus vitiis et virtutibus geschrieben. ${ }^{25}$

Die in der Salzburger Sammlung versammelten Texte wurden aus unterschiedlichen Vorlagen gewählt, die teilweise schon die Grundlagen für die Laienspiegel Alkuins und Paulinus gebildet haben. Zum einen wurden wie in einem Homiliar Predigten der Kirchenväter aufgenommen und überarbeitet, andererseits dienten Julianus Pomerius` De vita contemplativa, Pseudo-Basilius Admonitio ad filium spiritualem und Isidors Sententiae, aber auch Alkuins Schrift De virtutibus et vitiis selbst als Quellen für einige Texte. ${ }^{26}$

Mit hagiographischen Texten wurde noch ein anderes Genre einbezogen, das alternative Möglichkeiten zur Verdeutlichung von inhaltlichen Programmen bot. Gerade in Salzburg haben sich hagiographische Texte mehrfach als ideale Instrumente erwiesen, um unterschiedliche gesellschaftliche Themen $\mathrm{zu}$ artikulieren. ${ }^{27}$ Die unbestrittene Autorität eines Heiligen erschloss einen leichteren Zugang zu einem heterogenen Publikum und ermöglichte es, in Konfliktfällen sogar einfacher zu einem Kompromiss zu gelangen. Die Fixierung der Heiligenfeste im liturgischen Kirchenjahr förderte zudem eine regelmäßige (jährliche) Rezeption der einzelnen hagiographischen Texte. Vor allem aber gewährte das Zusammenspiel von Predigten und hagiographischen Texten einen größeren Spielraum, die moralischen Mahnungen dem Publikum zu unterbreiten. Während die Predigten die Tugenden und Sünden direkt ansprachen, ließen sich spezifische Aspekte mit den Passiones anschaulicher verdeutlichen. Alkuin sah den Wert der Märtyrer zur moralischen Erbauung des Grafen Wido ganz deutlich: „Wir können Märtyrer ohne Schwert und Flammen sein, wenn wir im Geiste wahrhaftig Geduld mit unseren Nächsten bewahren“. ${ }^{28}$ In diesem Sinn sollten auch die Texte der Salzburger Sammlung helfen, in Bayern ein neues moralisches Leitbild für die weltlichen Großen zu etablieren. Zusätzlich aber halfen die Passiones auch, die negativen Eigenschaften der römischen Amtsträger zu beleuchten. Es fehlte ihnen an Geduld, an Gerechtigkeit und Liebe, kurz an jenen Eigenschaften, die den christlichen Amtsträger auszeichnen sollten.

Dass die Wahl der in dieser Sammlung aufgenommenen Texte vor allem auf spätantike Passiones fiel und nicht auf bayerische bzw. fränkische Heiligenleben, lag daran, dass sie einen universelleren Rahmen boten und weniger mit der Geschichte einer gens verbunden waren. ${ }^{29}$ Das entsprach dem imperialen Programm in den ersten

24 Alkuin, Epistola 305, ed. Krusch 464. Vgl. Schmitz, Bonifatius und Alkuin 87.

25 Vita Alcuini 21 (ed. Wilhelm Arndt, MGH, SS, 15, 1, Hannover 1887) 182-197, hier 195.

26 Die meisten dieser Traktate bildeten schon die Grundlagen für die moralischen Texte Alkuins und des Patriarchen von Ravenna.

27 Vgl. Diesenberger, Gemeinschaft der Heiligen, passim.

28 Alkuin, De virtutibus et vitiis 9, ed. Migne 619: Sine ferro et flammis martyrum esse possumus, si patientiam veraciter in animo servamus cum proximis nostris.

29 Siehe den Vergleich zwischen der Passio Mauritii und der Vita Amandi. Siehe dazu Kap. 6. 
Jahren des 9. Jahrhunderts, das die Idee des Kaiserreichs über die einzelnen gentes setzte. Darüber hinaus waren spezifische Märtyrer und Heilige aber nicht nur mit diversen bischöflichen Kirchen und Klöstern verbunden, sondern auch mit lokalen Adelsgruppen. Der Märtyrer Laurentius hatte eine herausragende Rolle für die familia eines Deotold, ${ }^{30}$ der heilige Leodegar für die Sippe der Etichonen und der Bertolde, ${ }^{31}$ der Märtyrer Dionysius für die Waltriche um das Kloster Schäftlarn, ${ }^{32}$ der heilige Martin für die Familie eines Isancrim in Nörting und für die Mohingara, ${ }^{33}$ der Heilige Petrus für eine Familie an der Würm. ${ }^{34}$ In einer von Cotafrid bereits 737/738 gegründeten Marienkirche zu Enknach werden sogar Reliquien vom Grab des Herrn, der Maria, der Heiligen Peter und Paul, Andreas und Laurentius erwähnt. ${ }^{35}$ Für die meisten dieser Märtyrer und Heiligen finden sich sorgfältig kompilierte Texte in der Salzburger Sermones-Sammlung. Der „Moralisierung“ dieser spezifischen Erinnerungsressourcen für unterschiedliche soziale Gruppen, vom Heiligen eines Kirchenherren über einen Diözesanheiligen bis hin zu einem im Rang eines Reichsheiligen verehrten Martin, kam sicher ein großes Gewicht zu. Gerade weil vermittels der Passiones Fragen von Gerechtigkeit und Habgier angesprochen wurden, Aspekte, die für die Eliten von großer Bedeutung waren.

Der Vorteil einer Predigtsammlung zur Verbreitung der wichtigsten politischen Schlagworte ist evident: Im Rhythmus des liturgischen Kalenders werden diese den Rezipienten regelmäßig vorgetragen. Dass der Meineid eine der schlimmsten Sünden ist, wird nicht nur im gleichnamigen Sermo deutlich, sondern auch in den vorösterlichen Predigten, wenn die Gläubigen verstärkt zur Buße aufgefordert werden. Während in der Vorlage für eine dieser Predigten der spätantike Autor ein sinnenbetäubendes Panorama der Unsittlichkeiten entwarf, die man vermeiden sollte, wurden in der karolingischen Version nüchtern die verwerflichsten Sünden aufgezählt. Anstelle vom leidenschaftlichen Spielen, vom Schlemmen und Huren, vom Gebrauch von Parfums und wertvollem Schmuck treten prosaisch Hass und Mord. Aber noch vor diesen Todsünden wird der Meineid genannt. ${ }^{36}$

30 TF 167, ed. Bitterauf 164. Vgl. auch Diepolder, Altbayerische Laurentiuspatrozinien, passim.

31 Hartung, Bertolde in Bayern $144 \mathrm{f}$.

32 Störmer, Schäftlarn.

33 TF 447, ed. Bitterauf 382 f. Vgl. Störmer, Früher Adel 2, 369 f. Für die Familie der Mohingara vgl. TF 235, ed. Bitterauf 217.

34 TF 54, ed. Bitterauf 82.

35 TP 2, ed. Heuwieser 2 f. Vgl. Störmer, Früher Adel 2, 371.

36 Sermo 48: XLIII. Item alia unde supra. Victoris, ed. McCune: Iampridem te mundialis pompae oblectabat ambitio, et in servum te sibi concupiscentia saeculi vindicabat, nunc in luxuria pollutum, nunc in ebrietate dimersum, nunc in periurio perditum, nunc odio vel homicidio devastatum, ceterisque vitiis depravatum.Vgl.Victor Cartennae, De poenitentia liber unus, c. 18 (ed. Jacques Paul Migne, PL 17, Paris 1845) 979-1000, hier 991: Jampridem te mundialis pompae oblectabat ambitio, et in servum te sibi dominatus saeculi vindicabat, nunc in luxuria remissum, nunc in vinolentia demersum. Hi te modo exercitio aleonis intentum viderunt, alii ad ganeandum, alii in scortum projectum, isti lupanaribus man- 
Das Publikum für die Sammlung ist zunächst im Umfeld von Audulf, dem bayerischen Präfekten zu suchen, mit dem Arn von Salzburg am Beginn des 9. Jahrhunderts gemeinsam als missus tätig war. Einige Predigten der Sammlung, vor allem aber die am Ende eingefügten moralischen Texte, verdeutlichen, dass sie für einen Personenkreis geschrieben wurden, der mit Gerichtsangelegenheiten betraut war. Das betrifft Männer aus sehr unterschiedlichen sozialen Schichten. Hier könnte man an den iudex und späteren comes Orendil und an den Ostlandpräfekten Werner denken. Beide waren im Frühsommer 802 an jenem Gerichtstag anwesend, bei dem die Grundzüge des neuen Missaticums erläutert worden sein dürften. Wahrscheinlich wurden beide zu diesem Anlass überhaupt erst der bayerischen Öffentlichkeit in ihren neuen Funktionen präsentiert. Orendil machte sehr schnell Karriere und erwies sich sogar bei der Frage der Ansprüche seiner männlichen Nachkommen als ordentlicher Vertreter der Reformen. Werner aber übernahm eines der bedeutsamsten Ämter Bayerns als Grenzgraf des Ostlandes. 805 wurde er im Diedenhofer Kapitular nicht nur namentlich erwähnt, sondern er musste die darin formulierten Bestimmungen, etwa über das Leisten der Eide, auch durchsetzen. Zudem stammte er aus einer hochangesehenen Familie, der auch Wido von der Bretagne angehörte. Wenn Werner tatsächlich mit der Salzburger Sermones-Sammlung zu tun hatte, dann hörte er teilweise genau jene Texte, die seinem entfernten Verwandten von Alkuin persönlich auf den Leib geschneidert worden waren.

Die Predigten und Passiones konnten sowohl für die private Lektüre als auch für das Verlesen im kleineren Rahmen genutzt werden, wie es für die Homiliensammlung des Hrabanus Maurus für Lothar I. bezeugt ist. Wenn die Empfänger die Traktate vielleicht auch selbst nicht gelesen haben, so sollten sie vorgelesen werden. ${ }^{37}$ Das Hören solcher Texte unterschied sich dabei kaum von einer Predigt bzw. die Aufforderung zum Hören konnte durchaus auch auf die Predigt im Allgemeinen bezogen werden. ${ }^{38}$ Darüber hinaus eigneten sich die Texte aber auch für Mahnreden, wie sie um 800 von den missi und Grafen verlangt wurden. Der Gebrauch eines Sermo wie jener mit dem Titel De periurio ist durchaus als öffentlicher Vortrag im Rahmen einer Versammlung denkbar. Der Sermo De pace et concordia scheint sogar als Grundlage für Mahnreden bei zwei der wichtigsten Gerichtsversammlungen in Bayern in den ersten Jahren des 9. Jahrhunderts verwendet worden zu sein. Arn hatte in diesen Fällen die Initiative übernommen. Als weltliche Autorität stand aber der iudex Orendil den Verhandlungen vor. Es ist wahrscheinlich, dass Audulf selbst in seiner Funktion als missus mehrmals öffentliche Reden gehalten hat. Nicht zufällig hat Arn von Salzburg, sein langjähriger Berater und Kollege, die admonitiones Karls des Großen und das Aussenden der missi, die diese Mahnungen an jene weiterleiten sollten, die den Kaiser

cipatum, unguentis oblitum, aromatibus delibutum, et peregrinis cernebant oculis infectum. Splendebas vestibus, auro fulgebas, gemmis et pretiosissimis margaritis ornabaris.

37 Jonas, De institutione laicali, praefatio, 124c. Er solle den Text oft selber lesen bzw. sich vorlesen lassen.

38 So Noble, Secular sanctity 27. 
nicht persönlich aufsuchen konnten, in das semantische Umfeld der Predigt gestellt. Das Publikum der Sammlung war zunächst der Königsbote Audulf selbst, aber in weiterer Folge waren es auch die bayerischen Amtsträger, die anlässlich von Versammlungen zumindest Teile davon zu Gehör bekommen haben. Eine rasche Verbreitung der Sammlung in Bayern wurde aktiv und erfolgreich betrieben. Denn bis zur Mitte des 9. Jahrhunderts sind fünf meist unvollständig erhaltene Handschriften und Fragmente überliefert, deren Herkunft paläographisch meist nicht einer bekannten Schreibergruppe zuzuordnen ist. Wahrscheinlich dürften bis zur Mitte des 9. Jahrhunderts noch mehr Handschriften existiert haben, da es zum Teil doch große Varianten bei den Überlieferungsträgern gibt. Eine fast vollständige Kopie ist dem Freisinger Skriptorium zuzuweisen, eine weitere (unvollständige) wurde von einem presbyter Sandarat, der wahrscheinlich mit dem späteren archipresbyter desselben Namens gleichzusetzen ist, den Mönchen von Sankt Emmeram übereignet. Das betrifft allerdings nur den zweiten Teil der Sammlung, der um jene Texte verkürzt wurde, die für den monastischen Kontext weniger brauchbar waren. Zwei frühe pastorale Kompendien haben Teile der Sammlung übernommen und mit Texten aus Homiliaren ergänzt. Es ist gut denkbar, dass diese beiden Kompendien für einen Priester als Basis von Predigten an bayerische potentes vermittelt wurden.

Der Inhalt der Sammlung orientierte sich entlang mehrerer Leitlinien: an Fragen der Friedenssicherung, der Gerechtigkeit und der Unbestechlichkeit der Richter und an Themen der „Barmherzigkeit“ und „Fürsorgepflicht“ der potentes.

Dieser Hinwendung zum sozialen Handeln wurde zunächst aber eine eingehende Selbstprüfung zugrunde gelegt. Viele Predigten der Salzburger Sammlung setzen sich daher eingehend mit der Beichte und der Buße auseinander. Sie lenkten die Aufmerksamkeit auf das schuldhafte Verhalten des Individuums und auf die Notwendigkeit zur Einsicht und zur tätigen Buße: zum Fasten, Almosengeben und zur Nächstenliebe. Im kleinen Rahmen waren das überschaubare Handlungen, in größeren Zusammenhängen hatten sie aber mehr Gewicht. In den Notzeiten von 805 und 806 ordnete der Kaiser z. B. ein allgemeines dreitägiges Fasten und Almosengeben an, um Gott zu besänftigen. ${ }^{39}$ In Bayern ermahnte Arn in einer Synodalpredigt daher seine Hörer, diesen Anordnungen nachzukommen und er verschärfte sogar noch die Fastenauflagen für die Laien. ${ }^{40}$ Dabei schränkte er aber ein, dass dies nur erreicht werden könnte, wenn die Priester mit gutem Beispiel vorangingen und mit ihren Predigten Erfolg hätten. ${ }^{41}$ Die wiederholte Forderung zu diesen Bußauflagen in den Predigten war sicher eine Voraussetzung dafür, dass in Notzeiten auf Erlass des Kaisers hin solche Bestimmungen auch verlässlich durchgeführt werden konnten. Der in der Sermones-Sammlung hervorgehobene Aspekt des Beichtens hatte dabei eine ähnliche über den persönlichen Bereich hinausgehende Funktion. Der Kaiser verlangte von

39 Karoli ad Ghaerbaldum episcopum, ed. Boretius 245.

40 Arn von Salzburg, Synodalsermo, ed. Pokorny $392 \mathrm{f}$.

41 Arn von Salzburg, Synodalsermo, ed. Pokorny S. 391. 
seinen Untertanen, vor allem von seinen geistlichen und weltlichen Amtsträgern, die Offenlegung individueller Verfehlungen. 811 forderte er z. B. nicht nur von den Bischöfen und Äbten, dass jeder die Frage, ob er wirklich Christ ist, für sich beantworten und seine conversatio bewusst vor den anderen offenlegen solle, sondern auch von den comites. ${ }^{42}$ In einem an Bischöfe und Äbte adressierten Kapitular aus demselben Jahr wird der Aspekt der Selbstbeschau nochmals aufgenommen und wieder auf die Laien ausgeweitet. ${ }^{43}$

Diese Aufforderungen zur kritischen Beurteilung der eigenen Taten ähneln dem Gebot zur Selbstprüfung im Zusammenhang mit der Beichte. Mit der Aufwertung des Eides im Capitulare missorum von 802 wurde die individuelle Verpflichtung gegenüber dem Kaiser und gegenüber dem eigenen Seelenheil itensiviert und damit auch das Risiko erhöht, willentlich oder unwillentlich eidbrüchig zu werden. ${ }^{44}$ Die Metapher der Medizin, die im Zusammenhang mit der confessio und der paenitentia oft verwendet wurde, findet sich jedenfalls auch im Kontext der Befolgung kaiserlicher bzw. königlicher Vorgaben. In seiner Admonitio ermahnte Arn z. B. sein Publikum, dass allen kaiserlichen monita und statuta Folge geleistet werden müsste, da sie medicamenta nostrorum animarum seien, mit deren Hilfe man ewige Freuden erlangen könne. ${ }^{45}$

Jedem anderen inhaltlichen Schwerpunkt der Salzburger Predigten sollte daher eine eingehende Selbstprüfung vorausgehen. So auch den beiden Grundthemen: die Gerechtigkeit und die Friedenssicherung. Pax et concordia waren nicht nur ein bedeutsamer Leitgedanke im politischen Diskurs um 800, sondern auch ein wichtiges Thema in der Sermones-Sammlung. In der Überarbeitung einer Homilia des Beda Venerabilis wird die Versammlung der Jünger nach der Auferstehung Jesu hinter verschlossenen Türen zu einer Aufforderung umgedeutet, dass die Gläubigen in una fide in una pace et concordia in unaque spe et caritate untrennbar bleiben sollten. Der Sermo De pace et concordia, der die Sammlung beschließt, bietet viele Redewendungen zur Konsensbildung und wurde wahrscheinlich auch von den beiden missi in zumindest zwei Streitfällen als Vorlage für Mahnreden verwendet. Der Text diente auch als Vorlage für eine Bestimmung der Synode von Mainz im Jahr 813, bei der u. a. Arn von Salzburg den Vorsitz führte. Die Verwendung dieser Schlagworte in der SermonesSammlung und ihr Gebrauch im Rahmen von Versammlungen verdeutlicht, wie ein politischer Leitgedanke, der den offiziellen Diskurs am Beginn des 9. Jahrhunderts bestimmte, in lokale Gesellschaften getragen wurde.

Der Transfer solcher „Leitgedanken“ vom Hof in die Peripherie setzte allerdings die korrekten Abläufe jener sozialen Begegnungen voraus, in der sie zum Einsatz kommen sollten. Da die Präsenz der königlichen bzw. kaiserlichen Herrschaft vor allem bei lokalen Streitfällen sichtbar wurde, waren missi und Grafen angewiesen, auf die formale Richtigkeit der gerichtlichen Abläufe zu achten. Theodulf von Orléans

42 Capitula tractanda cum comitibus episcopis et abbatibus c. 9, ed. Boretius 161.

43 Capitula de causis cum episcopis et abbatibus tractandis c. 5, ed. Boretius 161.

44 Capitulare missorum generale a. 802, c. 4, ed. Boretius 92.

45 Arn von Salzburg, Admonitio, ed. Schneider 488. 
hatte nicht zuletzt mit seinem Fokus auf die iudices im Rahmen des Berichts seiner Visitationsreise als Königsbote die Rhetorik der Reform befeuert. Und Arn von Salzburg trug dazu bei, indem er auf die Kontrolle der weltlichen Amtsträger durch die Bischöfe pochte.

Ein wesentlicher Aspekt war die Eidleistung, die im Zuge des Inquisitionsverfahrens nun dazu diente, die Wahrheit in einem spezifischen Gerichtsfall ans Tageslicht zu befördern. Darüber hinaus berührte sie auch grundsätzlich die Frage nach der Loyalität zum König bzw. zum Kaiser. Viele Kapitularien, sowohl kaiserliche als auch bischöfliche, verurteilten den Meineid mit aller Schärfe. Theodulf von Orléans verlangte in seinem Ersten Kapitular, das wohl vor 813 entstanden ist, dass die Priester seiner Diözese dieses Thema in ihren Predigten unbedingt aufnehmen mussten. ${ }^{46}$ Die Bedeutung des Eides und die negativen Auswirkungen des Meineids bzw. des Eidbruchs wurden aber sicher auch während Gerichtsverhandlungen hervorgehoben, vor allem dann, wenn neue Formen der Vereidigung etabliert wurden. Arn und Audulf waren die ersten missi, die anlässlich einer bayerischen Gerichtsverhandlung in Freising die Vereidigung der Zeugen nach jener Form durchführten, die Karl der Große in seinem programmatischen Kapitular im Jahr 802 erlassen hatte. Mehrere Texte der Salzburger Sermones-Sammlung verurteilen den Meineid in einer Weise, die ihre Verwendung als Vorlage für Mahnreden vor der Vereidigung nahelegt.

Gleichzeitig sollten die mit den Gerichtsangelegenheiten betrauten Amtsträger selbst auf die moralischen Leitlinien ihres Amtes eingeschworen werden. Die Bestechlichkeit der Richter, das hat Theodulf aufgezeigt, führte nicht nur zu lokalen Missständen, sondern stellte die staatliche Ordnung grundsätzlich in Frage. Denn es waren genau jene missi und comites, und in den unteren Rängen iudices und ministri, die nicht nur Recht sprechen, sondern auch die Verordnungen und Anweisungen vom Hof im regionalen bzw. lokalen Kontext durchsetzen sollten. Nur die „Unbestechlichkeit“ der Amtsträger gewährleistete die ordnungsgemäße Durchführung der höfischen Vorgaben. Das umso mehr, als im Zuge der Reformen von 802/3 außergerichtliche Konfliktlösungen potentiell als Unrecht galten, den Gerichten und ihren Angehörigen also noch mehr politische Verantwortung zukamen.

Mit der Einführung des ständigen Missaticums in Bayern stellte das Inquisitionsbeweisverfahren eine alternative Suche nach der Wahrheit dar, die deutlich Effekte auf die in den Verfahren Beteiligten hatte. Denn in diesen Fällen wählte der missus die Zeugen aus und nicht die Streitparteien. Die Zeugen wurden einzeln verhört und wahrscheinlich auch belehrt. Zusätzlich erscheinen im Zuge der Schöffenverfassung auch neue Richter in den bayerischen Quellen, wie Orendil oder Kysalhard, die von den missi ausgewählt wurden und für die ein neues Anforderungsprofil galt. In der Salzburger Sammlung wurden daher die Themen der Unbestechlichkeit der Richter und die Rechtschaffenheit der Zeugen in mehreren Texten direkt angesprochen. Darüber hinaus wurde das Thema aber auch über die Sünde der cupiditas abgehandelt. Dieses 
Schlagwort findet sich in den Schlüsseltexten der Sammlung, wie in dem den Jahreskreis abschließenden Sermo De laude caritatis, der auf einem gleichnamigen Kapitel von Julianus Pomerius De vita contemplativa basiert. Deutlicher als in der Vorlage ist die Habgier in der Salzburger Version die stärkste Gegenkraft der caritas. ${ }^{47}$ Die Habgier ist aber auch zentrales Thema in den Passiones, die den Rezipienten der Texte eindringlich ihre negativen Auswirkungen verdeutlichten. Ein Vergleich mit den römischen Amtsträgern, die im Zuge der Christenverfolgungen nach dem Vermögen der Märtyrer trachteten, sollte den bayerischen potentes als negatives Spiegelbild dienen, sich nicht am Besitz der Schwächeren zu vergreifen. Denn die Habgier der römischen Richter verführte sie zu Zorn und Wut, zwei Todsünden, und in weiterer Folge zur grausamen Misshandlung und Ermordung von Christen. Schon Paulinus von Aquileia hatte zu den negativen Auswirkungen der Habgier Mord und Totschlag gezählt. ${ }^{48}$ Aus diesem Grund wird die grausame Misshandlung der Christen in den Passiones besonders detailliert geschildert. Die Grausamkeit dabei ist Sinnbild für all jene Verfehlungen, die ein unsittliches Leben ausmachten.

In einem größeren Kontext hatten cupiditas und avaritia auch schlechte Auswirkungen auf eine für alle Bevölkerungsteile ausreichende Verteilung ökonomischer Güter. Im Jahr 806 sah Karl der Große angesichts einer Hungersnot die Notwendigkeit, in einem Kapitular den populus auf die cupiditas, die avaritia und concupiscentia hinzuweisen, also auf jene Todsünden, die in zahllosen Predigten dieser Zeit verurteilt wurden und deren verheerende Auswirkungen nun zu beobachten waren. Diese Ermahnung des Kaisers, sich auf die rechten Werte zu besinnen, verdeutlicht, dass die eher abstrakten Themen der Predigten durchaus ihren Sitz im Leben hatten. Der Eigennutz der Reicheren und der Mangel an Solidarität gegenüber ihren Mitmenschen zeitigten in diesen Krisenzeiten eben auch spürbar (ökonomische?) Effekte auf die Versorgung der Bevölkerung. Darüber hinaus wurde der Kaiser auch des mangelnden Bewusstseins der regionalen Eliten gewahr, in Notzeiten selbst aktiv zu werden und nicht auf Befehle von oben zu warten. ${ }^{49}$ Daher sollte das Verständnis für die moralische Verantwortung für die Mitmenschen unter den potentes langfristig besser verankert werden. Dafür eigneten sich besonders Predigten. Denn sie verdammten nicht nur regelmäßig den Geiz und die Habgier, sondern boten auch ein vielgestaltiges Modell von „Gabe und Gegengabe“ an, das den Individuen ein friedliches Zusammenleben ermöglichen sollte. An der Spitze der guten Taten stand die tätige Barmherzigkeit. Nur das Almosengeben (elemosyna) stellte schon in den Augen Theodulfs von Orléans ein geeignetes Mittel gegen das Laster der Habsucht (avaritia) dar, und die meisten Prediger folgten ihm darin. ${ }^{50}$ Dabei war das Almosengeben ein weit gespannter Begriff, der die sonntäglichen Opfergaben am Altar für die Armen ebenso

47 Sermo II, 46: De laude caritatis ; vgl. Julianus Pomerius, De vita contemplativa 3, 12, ed. Migne 415 - 520, hier 493.

48 Paulinus von Aquileia, Liber exhortationis c. 53, ed. Migne 260.

49 Vgl. schon: Capitulare missorum in Theodonis villa c. 4, ed. Boretius 122.

50 Vgl. Libri Carolini 2, 30, ed. Freeman 96. 
umfasste wie die Schenkung von Land oder die Huld des Herrschers. In zahlreichen Predigten und Kapitularien wurde den potentes die Fürsorge für die Bedürftigen ans Herz gelegt und auf die himmlische Entlohnung für diese gute Tat verwiesen. Im Zug des großen programmatischen Kapitulars von 802 hat Karl der Große sogar seine persönliche Aussicht auf die Belohnung im ewigen Leben mit dem guten Willen aller seiner Bischöfe, Äbte und comites verbunden, die Armen, Witwen, Waisen und peregrini aufzunehmen und zu schützen. ${ }^{51}$ Damit wären die potentes dazu verpflichtet gewesen, ihre Barmherzigkeit und ihre Fürsorge für die Schwächeren unter Beweis zu stellen. Tatsächlich beeinträchtige die Habgier der potentes oft genug auch staatliche Interessen. ${ }^{52} \mathrm{Ob}$ aber die in den Kapitularien geforderten Maßnahmen tatsächlich wirtschaftliche Effekte bewirkten, ist nicht festzustellen.

Eine finanzielle oder statusbezogene Aufwertung der Bedürftigen stand ohnehin nicht zur Debatte. Die Aufmerksamkeit des Frankenherrschers galt den sozial schwächeren Freien, die er vor den Übergriffen der potentiores schützen wollte. Daher wurde vielmehr die Stabilisierung der sozialen Verhältnisse angestrebt, in denen auch Bedürftigere überleben konnten. Der Begriff pauper bezeichnete nicht unbedingt eine mittellose Person, sondern wurde kontextabhängig verwendet. Ausdrücklich wird in der von der Admonitio generalis inspirierten Predigtsammlung des Pseudo-Bonifatius die Wahrung der sozialen Hierarchien als unabdingbare Voraussetzung zur Erlösung des Einzelnen gefordert. ${ }^{53}$ Auch Alkuin, die bedeutendste moralische Autorität am Hof Karls des Großen, beobachtete zwar um 800 die Auswirkung einer Hungerkatastrophe unter den Unfreien von Tours, die, wie er schreibt, so vor der Not flohen, wie die pauperes vor einem Steuereintreiber (exactor). ${ }^{54}$ Er selbst litt aber offenbar unter keinem Mangel und kommentierte das Ereignis eher nüchtern, als Mitleid mit den Betroffenen zu zeigen. Diese distanzierte Haltung zum Unglück der Mittellosen zeigt sich auch in einem Zitat aus De virtutibus et vitiis. Die pauperes, die zu Unrecht unterdrückt werden, würden sehr schnell das irdische Unglück beenden, meint er dort. Ihre Unterdrücker aber würden den ewigen Flammen überantwortet werden. ${ }^{55}$ Von diesem Geist wurde nicht nur Alkuins Traktat getragen, sondern auch die Salzburger Sermones-Sammlung, in der diese Passage Aufnahme fand. ${ }^{56}$

Die pauperes als Empfänger der Almosen wurden in vielen Predigten nämlich hauptsächlich als Gegenbild zu den potentes aufgebaut, das grundsätzlich dazu genutzt wurde, diese zum tugendreichen Leben zu ermahnen und auf Verfehlungen

51 Capitulare missorum generale c. 14, ed. Boretius 94.

52 Vgl. z. B. Capitulare missorum in Theodonis villa c. 16, ed. Boretius 125.

53 Siehe auch Missi cuiusdam admonitio, ed. Boretius $239 \mathrm{f}$.

54 Alkuin, Carmen 8, ed. Dümmler 228, Z. 11-13.

55 Alkuin, De virtutibus et vitiis 21, ed. Migne col. 269: Magis dolendi sunt qui opprimunt pauperes, quam qui patiuntur iniuriam. Illi enim qui opprimuntur, temporalem miseriam cito finiunt: illi uero qui opprimunt eos per iniustitiam, aeternis flammis deputantur.

56 Mit Ausnahme von Jonas ist das keine Debatte in den Fürstenspiegel, und auch nicht für die Sermones, falls diese überhaupt auf Unfreie eingehen. Vgl. Stone, Morality and Masculinity 188. 
aufmerksam zu machen. Dasselbe ist für die Kapitularien festzustellen, wo die „Armen“ oft erst dann als eine moralische Kategorie aktualisiert wurden, wenn die Eliten ermahnt und zum rechten Verhalten zurückgeführt werden sollten. Die Aufforderung zur „Fürsorge für die Armen“ diente damit auch zur Festigung jener Autorität, die sie (angeblich) übernommen hatte. Insofern drückte die Bezeichnung des fränkischen Kaisers als pater pauperum nicht nur seine moralische Superiorität aus, sondern auch seine herrschaftliche.

Die Rhetorik der Habgier und des Almosengebens, die in Predigten und Kapitularien gepflegt wurde, bestimmte auch die Fragen der Sicherung und Transfers von Besitz. Denn als Almosen konnten schließlich auch Übertragungen von Landbesitz an die Kirche verstanden werden. Es war nicht zuletzt der Streit um Land und Besitzrechte, der die kaiserlichen missi in Bayern in den ersten Jahren des 9. Jahrhunderts hauptsächlich beschäftigte. Die Zurücksetzung erbberechtigter Söhne hatte zu vermehrten Konflikten mit den begünstigten Kirchen geführt. Zusätzlich waren einige Adelsgruppen im Loyalitätskonflikt mit den Karolingern und wurden enteignet. Neue Amtsträger versuchten sich auf Kosten anderer in Bayern festzusetzen. Die Ansprüche der zurückgesetzten Erben wurden nun mit Hilfe der Predigten in den Kategorien der Habgier und des Almosengebens erfasst. Die mit großer Härte geführten Auseinandersetzungen gingen meist zuungunsten der tatsächlich benachteiligten Adelssöhne aus. Im günstigsten Fall strichen sie ihre Fahnen und erwiesen sich so als großzügige Stifter von Almosen. Im schlechtesten Fall wurden sie als habgierig bezeichnet und mussten erst recht von ihren Ansprüchen Abstand nehmen.

Für jene potentes, die aber die Armen mit Almosen unterstützen und so ihre Barmherzigkeit übten, gab es nicht nur himmlischen Lohn zu gewinnen, sondern auch symbolisches Kapital, im Idealfall sogar das Wohlwollen des Hofes. Das waren wichtige Voraussetzungen, um sich bei ökonomischen Verhandlungen größeren Umfangs durchsetzen zu können. Denn jene Amts- und Würdenträger, die im Kleinen ihre Barmherzigkeit unter Beweis gestellt hatten, konnten im Zusammenhang größerer Gütertransfers und der damit verbundenen Verhandlungen zumindest weniger leicht der Habgier bezichtigt werden als andere, die über einen schlechteren Ruf verfügten. Eine Reihe von Besitzübertragungen an Freising im Jahr 827 zeigt den Ablauf solcher Transfers und verdeutlicht die entscheidende Rolle der bischöflichen Predigt in diesem Zusammenhang.

An dieser Stelle zeigt sich aber ein anderer Aspekt: Die in den Predigten und Kapitularien angelegten moralischen Werte konnten erst recht zum Missbrauch führen. Karl der Große hatte 811 mit großer Sorge bemerkt, dass geistliche Herren „durch die Verheißung der Glückseligkeit im Himmelreich“ bzw. „durch Androhung der ewigen Höllenpein“ versuchten, jene zu übervorteilen, die sie eigentlich zur Tugend mahnen, ja denen sie selbst sogar als Vorbilder dienen sollten. Und selbst die moralische Evaluierung innerhalb der Eliten stand auf dem Spiel, wenn die Zuwendung zu den Armen nur noch als taktisches Element begriffen wurde, um am Hof symbolisches Kapital zu sammeln, wie es etwa Hitto von Freising tat. In beiden Fällen hatte sich die rhetorische und moralische Ausbildung der Kleriker ins Gegenteil verkehrt. Die Predigt 
erwies sich eben als das zweischneidige Schwert, von dem Alkuin in seinen Schriften gesprochen hatte. Zudem wurden die moralischen Standards nun durchaus auch dazu gebraucht, sich gegenseitig öffentlich sündiges Verhalten vorzuwerfen. Falsche Beschuldigungen wegen unmoralischen Verhaltens von anderen zählten zu den unangenehmen aber nicht zu verhindernden Seiten eines herrschaftlich gestützten moralischen Diskurses. ${ }^{57}$

Nur in Ausnahmefällen gingen schriftlich niedergelegte Predigten vom Beginn des 9. Jahrhunderts auf reale Ereignisse ein. Das sollte ihren Wert für die historische Analyse aber nicht schmälern. Im Idealfall verfestigte die Predigt moralische Grundwerte in allen Regionen und in allen Gesellschaftsschichten des Reiches. Sie griff speziell auf Amtsträger zu und bot Elemente zu ihrer Kontrolle oder zur Selbstevaluation. Sie stabilisierte soziale Verhältnisse und regulierte juristische Abläufe. Sie vermittelte Modelle des Gebens und der Friedenssicherung. Kurz: Sie war ein ideales Medium, das die Politik des Herrschers entscheidend vorantreiben und dem sozialen Zusammenhalt seines Reiches dienen konnte.

Aber die Realität zeigte, dass das soziale Leben nicht entlang dieser moralischen Linien verlief. In der Regel verhielt sich wohl kaum jemand nach den in diesen Texten skizzierten Grundsätzen. Neben jenen Grafen wie Orendil, die sich der Rhetorik der Reform verpflichtet fühlten, gab es andere, wie den jüngeren Coteram, der auch unter der Aufsicht Arns und Audulfs rücksichtslos seine Interessen durchzusetzen trachtete.

Und selbst die Kommunikationskanäle, die überhaupt erst die Vermittlung dieser Inhalte gewährleisten sollten, konnten meist nicht so frei gehalten werden, wie erhofft. In dem von missi für comites im Jahr 806 formulierten Kapitular zeigt sich deutlich das Grundproblem der Kontrolle von lokalen Gesellschaften: Die Menschen wollten ihre Angelegenheiten unter sich ausmachen, und wie im konkreten Fall nicht vor das Gericht eines missus bringen. ${ }^{58}$ Nur unter höchstem Druck verliefen soziale Begegnungen nach den moralischen Standards, die in den Predigten und Mahnreden definiert wurden. So etwa als Arn von Salzburg noch als missus in Bayern tätig war und nachweislich gerichtliche Entscheidungen erzwingen konnte.

Trotzdem hatten diese Texte einen unschätzbaren Wert. Sie stellten ein vom Hof propagiertes oder zumindest von dort inspiriertes diskursives Repertoire moralischer Leitlinien zur Verfügung, das in der Umgebung, in die es getragen wurde, zumindest

57 Eric J. Goldberg, Dominus Hludowicus Serenissimus Imperator sedens pro tribunali: Conflict, Justice, and Ideology at the Court of Louis the German, in: Streit am Hof im frühen Mittelalter, ed. Matthias Becher/Alheydis Plassmann (Göttingen 2011) 175-202, hier 183, über Ludwig den Deutschen, der das Vermögen eines angeblichen untreuen Alemannengrafen einzieht und es später wieder zurückgibt, nachdem er bemerkt, dass er bösen Gerüchten Glauben geschenkt hat.

58 Capitula a missis dominicis ad comites directa c. 5, ed. Boretius 185: Deinde observate etiam valde, ne aut vos ipsi aut aliquis, quantum vos praevidere potestis, in vestro ministerio in hoc malo ingenio deprehensus fiat, ut dicatis: 'tacete, donec illi missi transeant, et postea faciamus nobis invicem iustitias!' et per illam occasionem ipsae iustitiae aut remaneant aut certe tarde fiant; sed magis certate, ut ante factae fiant quam nos veniamus ad vos. 
jene Themen anzeigte, die dem politischen Willen des Herrschers entsprachen. Wo der missus keinen Zugriff hatte, konnte die Predigt vielleicht noch etwas erreichen. Diese Texte bestimmten die Art und Weise, wie über Gesellschaft bzw. über gesellschaftliche Begegnungen gesprochen werden sollte. Das konnte im Alltag durchaus ignoriert werden. Aber in spezifischen Situationen kamen Elemente dieses Repertoires unvermutet zum Tragen. In einer Welt, in der grundsätzlich jeder nahm, was er bekommen konnte, wurde dem im Fallen begriffenen Matfrid von Orléans plötzlich Korruption zum Vorwurf gemacht, ohne dass er sich dieser Sünde tatsächlich schuldig gemacht hatte. ${ }^{59}$ Das war besonders bitter, denn von Jonas hatte er das dreibändige Werk De institutione laicali erhalten, das die moralischen Standards dieser Zeit nicht nur wiedergab, sondern auch wesentlich mitbestimmte. ${ }^{60}$ Sein Beispiel zeigt, dass ein jeder potens die Moral der Predigten eben doch zu fürchten hatte. Es gab sicher wirkungslosere Herrschaftsinstrumente. Letztlich sorgte sich ein Christ doch darum, seine Seele zu retten und auch für Karl den Großen lag der Wert der Predigt darin, die Rettung seiner eigenen Seele und jene seiner Untertanten sicherzustellen, für die er verantwortlich war. Das legitimierte ihn zugleich, über die Moralisierung seiner Politik auf lokale Gesellschaften zuzugreifen und den Zusammenhalt seines Reiches zu festigen. In seiner Gegenwart erwies er sich damit als umsichtiger, erfolgreicher Herrscher, für seine Zukunft aber als ein praedicator, der dem populus Christi den Weg in das himmlische Königreich wies.

59 Le Jan, Justice royale 59.

60 Teile des Textes wurden sogar in die Konzilsakten von 829 übernommen. Vgl. auch die Aufforderung Agobards, Matfrid möge sein Verhalten und das seiner Untergebenen bessern, damit sie die besten ministri Gottes und adiutores des Kaisers seien. Damit war auch gemeint, dass er Betrug auslöschen sollte. Vgl. Agobard von Lyon, Epistola 10, ed. Dümmler $202 \mathrm{f}$. 
